\title{
Integrated palliative care in the Spanish context: a systematic review of the literature
}

Eduardo Garralda ${ }^{1,5^{*}}$, Jeroen Hasselaar ${ }^{2}$, José Miguel Carrasco ${ }^{1,5}$, Karen Van Beek ${ }^{3}$, Naouma Siouta ${ }^{3}$, Agnes Csikos $^{4}$, Johan Menten ${ }^{3}$ and Carlos Centeno ${ }^{1,5}$

\begin{abstract}
Background: Integrated palliative care (IPC) involves bringing together administrative, organisational, clinical and service aspects in order to achieve continuity of care between all actors involved in the care network of patients receiving palliative care $(P C)$ services. The purpose of this study is to identify literature on IPC in the Spanish context, either in cancer or other advanced chronic diseases.

Methods: Systematic review of the literature about IPC published in Spain between 1995 and 2013. Sources searched included PubMed, Cochrane Library, Cinahl, the national palliative care Journal (Medicina Paliativa), and Google. Evidence on IPC in care models, pathways, guidelines and other relevant documents were searched. Additionally, data were included from expert sources. Elements of IPC were considered based on the definition of IPC and the Emmanuel's IPC tool. The main inclusion criterion was a comprehensive description of PC integration.

Results: Out of a total of 2,416 titles screened, 49 were included. We found two models describing IPC interventions achieving continuity and appropriateness of care as a result, 12 guidelines or pathways (most of them with a general approach including cancer and non-cancer and showing a theoretical IPC inclusion as measured by Emmanuel's tool) and 35 other significant documents as for their context relevance (17 health strategy documents, 14 analytical studies and 4 descriptive documents). These last documents comprised respectively: regional and national plans with an IPC inclusion evidence, studies focused on IPC into primary care and resource utilisation; and descriptions of fruitful collaboration programmes between PC teams and oncology departments.

Conclusions: The results show that explications of IPC in the Spanish literature exist, but that there is insufficient evidence of its impact in clinical practice. This review may be of interest for Spanish-speaking countries and for others seeking to know the status of IPC in the literature in their home nations.
\end{abstract}

Keywords: Integration, Palliative Care, Spain, Model, Guideline, Pathway

\section{Background}

The European Union is experiencing an acute ageing of the population in recent decades with growing numbers of patients suffering from cancer and non-cancer disease [1]. Spain is also confronted with this development and has to develop palliative care (PC) services in response, aiming to improve the quality of life for patients and their families facing life-threatening illness.

\footnotetext{
* Correspondence: egarralda@unav.es

${ }^{1}$ Atlantes Research Programme, Institute for Culture and Society, University of Navarra, Campus Universitario, 31009 Pamplona, Navarra, Spain

${ }^{5}$ Instituto de investigación sanitaria de Navarra (IdiSNA), Pamplona, Navarra, Spain
}

Full list of author information is available at the end of the article
In order to handle complex care situations, a sustained, expert and quality care provision is needed. Continuity of care is essential for patients with complex needs and engagement with a variety of service providers. In order to achieve this, it is necessary to integrate $\mathrm{PC}$ at all care levels and across interprofessional team and agency boundaries [2].

The challenge of integrating PC into the health system, at different care levels, for cancer and non-cancer, has already been acknowledged in the PC Strategy of the 2007 National Health System of Spain. It was estimated that 380.000 people die in Spain every year and that 
between 50 and $60 \%$ of these may need PC in the last stage of their illness. The Strategy clearly states the need for coordinated action between diverse health providers to guarantee continuity of care, the timely identification of patients with PC needs, evidence of care planning that addresses the particular needs of patient and family caregivers, the provision of appropriate services, and ongoing assessment systems [3].

This paper attempts to identify integration of $\mathrm{PC}$ in administrative, organisational, health services and clinical documents in the Spanish literature, either in cancer or other advanced chronic disease.

\section{Methods}

A systematic review following PRISMA guidance [4] was conducted including scientific and grey literature. IPC has been defined as the administrative, organisational, clinical and service aspects in order to achieve continuity of care between all actors involved in the care network of patients receiving $\mathrm{PC}$ [5].

\section{Search strategy}

Five different sources were searched: 1) PubMed database, including $\mathrm{MeSH}$ and free text terms (Table 1); 2) The Cochrane library database (Table 2); 3) The Cinahl database (Table 3); 4) The only PC Spanish journal "Medicina Paliativa" ("Palliative Medicine") (manually searched); 5) Google (www.google.com) with (Table 4); and 5) Experts from different national Spanish societies of medicine (neurology, pulmonology, nephrology, geriatrics, cardiology and medical oncology,) were sent a letter to recommend experts on integrated care that could suggest studies on the topic in Spain.

\section{Search criteria}

The search period dated from January 1995 (based on the publication year of the Calman-Hine report [6] which constitutes the first national cancer plan in Europe) to December 2013. Documents regarding interventions aimed at children, integrated mechanisms only focusing

Table 1 PUBMED search terms and chain

(Spain[MeSH Terms]) AND (((hospices OR supportive care OR supportive care OR end of life care OR palliative OR palliative care [MeSH Terms] OR hospice* OR terminal care OR coordinated care OR integrated care OR transmural care OR progressive patient care) AND ("end stage disease" OR end stage disease* OR dying OR death [MeSH Terms] OR Chronic disease [MeSH Terms] OR Chronic disease* OR terminally ill* OR terminally ill [MeSH Terms] OR cancer) AND (care pathway* OR care pathway OR pathway* OR patient transfer* OR patient transfer OR patient care team OR managed care program* OR continuity of patient care OR patient care management OR patient care plan* OR patient care planning OR illness trajectory OR "advanced care planning" OR advanced care planning OR delivery of health care OR models of care OR model of care OR model organizational OR models organizational OR organizational model* OR guideline*) NOT ((birth) OR child) OR pediatrics)) NOT ((animals[mh] NOT humans[mh])))
Table 2 COCHRANE library search terms and chain

((palliative medicine) or (palliative care)) and ((guideline) or (pathway) or (model) or (plan) or (programme))and ((integrate) or (integrated) or (integrative) or (integration)) and (Spain)

((medicina paliativa) or (cuidados paliativos)) and ((guía) or (vía) or (modelo) or (plan) or (programa))and ((integrar) or (integrado) or (integrativo) or (integración)) and (España)

on the terminal phase (imminent death) and opinion in clinical case reports and editorial letters were excluded.

\section{Data selection}

We concentrated on evidence addressing models, guidelines and pathways in IPC in cancer and chronic advanced disease. Models were considered to be "project models" implemented in a particular setting and describing an effective integration of PC; guidelines were those defined by the AGREE instrument as systematically developed statements to assist practitioner and patient decisions about appropriate health care for specific clinical circumstances [7]; and care pathways were considered to be complex interventions designed for mutual decision making and the organisation of care processes for a well-defined group of patients during a well-defined period [8].

Secondly, we searched documents related to strategy or of description and evidence of IPC. These included strategic documents (National or Regional public plans promoting and pointing out the need for IPC), descriptive documents (theoretical integrative programmes, models and general situation of PC) and analytical studies (observational or experimental studies assessing an experience or IPC intervention but different to models in terms of their level of effectiveness and their score in the Hawker et al. tool [9]).

All identified documents were downloaded into a database including the title, abstract and summary or introduction (depending on the type of document). Two researchers separately selected material for the data extraction phase (disparities were resolved by consensus). Only selected documents were screened for full text revision and data extraction.

\section{Data extraction}

The data extraction form included: title, authors and collaborators, type of document (model, guideline, pathway

Table 3 CINAHL database search terms and chain ((palliative medicine) or (palliative care)) and ((guideline) or (pathway) or (model) or (plan) or (programme))and ((integrate) or (integrated) or (integrative) or (integration)) and (Spain)

((medicina paliativa) or (cuidados paliativos)) and ((guía) or (vía) or (modelo) or (plan) or (programa))and ((integrar) or (integrado) or (integrativo) or (integración)) and (España) 
Table 4 GOOGLE search terms and chain

guía paliativos OR programa oncología OR cáncer OR neurología OR neumología OR nefrología OR cardiología OR respiratoria OR cardíaca OR renal OR neurológica OR demencia "paliativo OR terminal" filetype:pdf

or other), date, setting, and type of disease, participants' demographics, study design, intervention, setting, outcome measures, results and quality assessment according to Hawker et al. [9]. The protocol of the Hawker et al. appraisal tool [9] rates the following aspects from 1 (very poor) to 4 (good): "1) Abstract and title: Did they provide a clear description of the study?; 2) Introduction and aims: Was there a good background and clear statement of the aims of the research?; 3) Method and data: Is the method appropriate and clearly explained?; 4) Sampling: Was the sampling strategy appropriate to address the aims?; 5) Data analysis: Was the description of the data analysis sufficiently rigorous?; 6) Ethics and bias: Have ethical issues been addressed, and what has necessary ethical approval gained? Has the relationship between researchers and participants been adequately considered? 7) Results: Is there a clear statement of the findings?; 8) Transferability or generalizability: Are the findings of this study transferable (generalizable) to a wider population?; 9) Implications and usefulness: How important are these findings to policy and practice?"

\section{Content assessment}

Specific information relating to pathways and guidelines such as description of the document, inflection point (prognosis), and the presence of a separate reference to another guideline or pathway, were included in the database. Studies were included that met two or more of Emmanuel's criteria as agreed by the InSup-C Consortium for its completeness of the IPC content [10]. This tool is comprised of different indicators rating the level of PC integration and explaining how is it being integrated. It proposes 11 specific criteria: "discussion of illness limitations and prognosis; recommendations for conducting a whole patient assessment including the patient's physical, social, psychological and spiritual issues, their family and community setting; recommendations for when to review assessments; recommendations for when PC should be integrated; assessment of the patient's goals for care; continuous goal adjustment as the illness and the person's disease progresses; PC interventions to reduce suffering; advance care planning; recommendations on involving a PC team; recommendations on PC at the last moments of life; and recommendations on grief and bereavement."

\section{Quality assessment}

A quality assessment process agreed by the project research team was made to determine the quality of the guidelines and pathways according to the manner in which they were developed: systematic review, consensus methods, evidence based and quality assessment; systematic review and consensus methods; systematic review only; consensus methods only; unclear methods; and other options. Data from descriptive and analytic studies also underwent a quality evaluation using the Hawker et al. critical appraisal tool [9].

\section{Ethical approval}

Ethical approval was not required as all the information is already published and human beings were not involved.

\section{Results}

After duplicates were removed, 2,416 documents were identified: PubMed 587 articles, Cochrane 2 documents, Cinahl 5 documents, the National Journal "Medicina Paliativa" ("Palliative Medicine") 1020 articles, Google 800 documents, and two documents suggested by experts. After title and abstract screening 2329 records were excluded and after full text revision 49 documents were included for data extraction (Fig. 1).

In our review, two empirical models of IPC (Table 5), 12 guidelines and pathways in cancer and nonmalignant diseases (Table 6) and 35 documents providing significant information relating to the Spanish context were identified (Table 7).

\section{Models}

Two models were identified: "Atención a pacientes crónicos avanzados no oncológicos con necesidad de cuidados al final de la vida en un hospital de media y larga estancia" (End-of-life care of advanced chronic noncancer patients in a medium and long term hospital) [11] and the document "Influencia del Plan Integral de Cuidados Paliativos de la Comunidad de Madrid” (Influence of the Integrated Plan of Palliative Care of the Autonomous Community of Madrid in the medical activity of a hospital based palliative care unit) [12].

These two models, both published in 2011 and applying to hospital settings, achieved a high quality score (Hawker et al.,) [9]. Both were observational studies, the first of which addressed non-cancer patients suffering from advanced chronic disease, identified and evaluated through an exhaustive multidimensional study and interdisciplinary teamwork in a long stay hospital delivering end-of-life care [11]. The second, considered both cancer and non-cancer disease and concluded that the integration of a Home Care team within a PC unit improves continuity of care and coordination between levels of healthcare [12] (Table 5). 


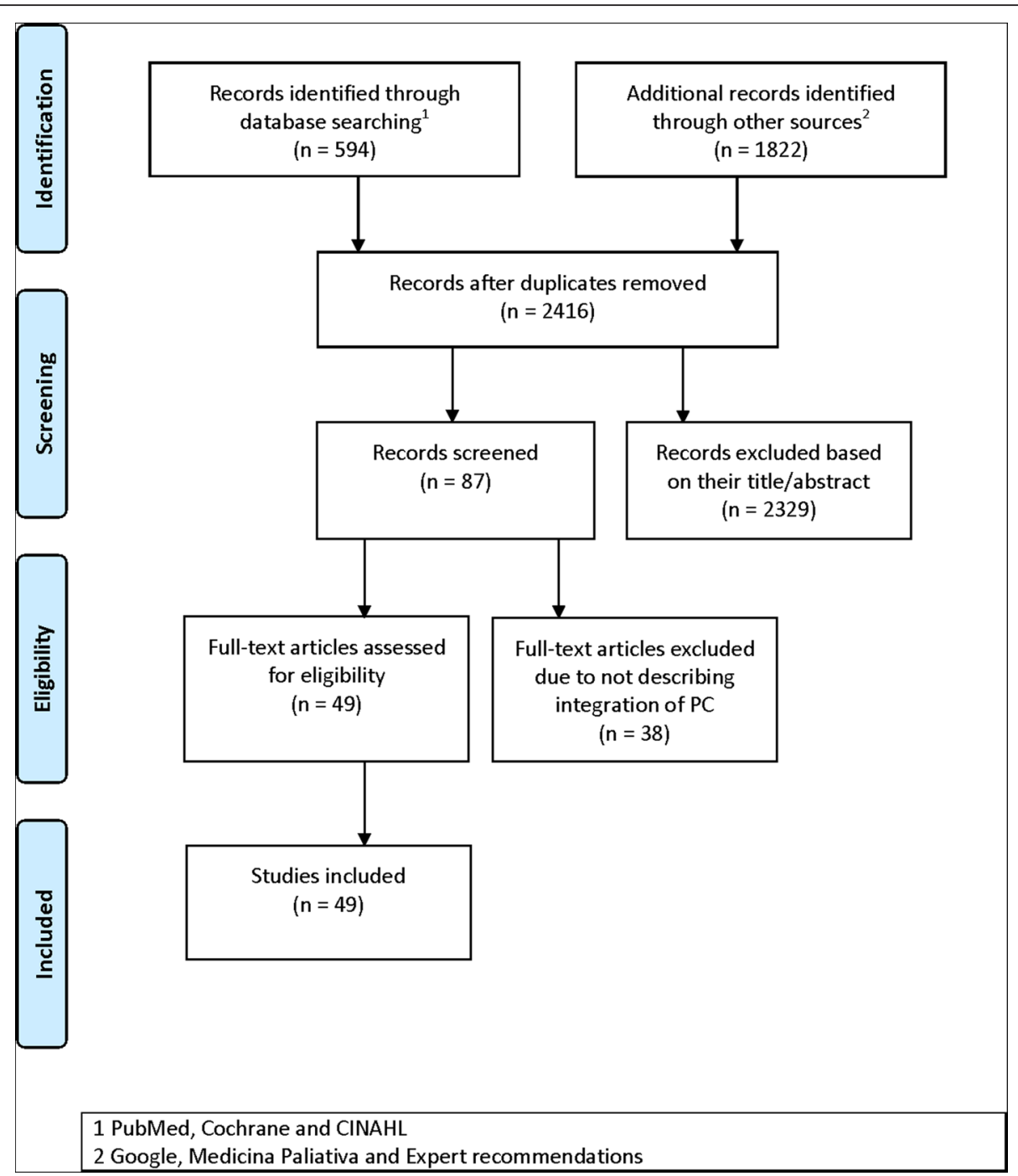

Fig. 1 Flow diagram of the systematic review (modified from Moher et al. (2009))

\section{Guidelines and pathways}

\section{Guidelines and pathways on both malignant} and non-malignant diseases

Four clinical guidelines for cancer and non-cancer were identified. The "Guía de Práctica Clínica sobre Cuidados Paliativos" (Clinical Practice Guideline on Palliative Care) [13], published in 2008, matched 10 out of 11 Emmanuel criteria [10]. This guideline is in line with the other three identified in that they all included recommendations about PC interventions to reduce suffering [14-16]. Except for "Guía de Cuidados Paliativos" (Palliative Care Guideline), the other three concurred in three other criteria: discussion of illness limitations and prognosis; recommendations for conducting a whole patient assessment including their family and their community setting; and recommendations on when PC should be integrated (Table 6).
Three pathways in cancer and non-cancer were found. One pathway referred to out-of-hospital emergencies [17], another related to home settings [18], and finally, one applied to both [19]. The three pathways contain recommendations on when PC should be integrated; suggestions to intervene to reduce suffering as needed; and recommendations on care during the last hours of living (Table 6).

\section{Guidelines and pathways on cancer}

One clinical guideline, "Guía de recomendaciones clínicas: cancer colorectal" (Guideline of clinical recommendations: colon cancer) [20] and one pathway, "Cuidados paliativos en el enfermo oncológico, documentos para la gestión integrada de procesos asistenciales relacionados con el cáncer, Proyecto Oncoguías" (Palliative care in the oncologic patient, documents for integrated management of care processes related to cancer, Oncoguías Project) [21], 
Table 5 Models of integrated Palliative Care $(n=2)$

\begin{tabular}{|c|c|c|c|c|c|c|}
\hline First author, and Year & Disease & Design & $\begin{array}{l}\text { Quality } \\
\text { assessment } \\
\text { according to } \\
\text { Hawker et al. }\end{array}$ & Intervention & Outcome measurements & $\begin{array}{l}\text { Results/effectiveness of } \\
\text { intervention }\end{array}$ \\
\hline Vicente et al., 2010. [12] & $\begin{array}{l}\text { Malignant and Non-malignant } \\
\text { Disease }\end{array}$ & $\begin{array}{l}\text { Retrospective } \\
\text { and } \\
\text { prospective } \\
\text { cohort study }\end{array}$ & 30 & $\begin{array}{l}\text { Influence of the } \\
\text { Integrated Plan of } \mathrm{PC}^{\mathrm{a}} \text { of } \\
\text { the Autonomous } \\
\text { Community of Madrid in } \\
\text { the medical activity of a } \\
\text { hospital based } \mathrm{PC}^{\mathrm{a}} \text { unit. }\end{array}$ & $\begin{array}{l}\text { Improvement in } \\
\text { continuity of care, } \\
\text { coordination amongst } \\
\text { assistant bodies, increase } \\
\text { in mean stay at the } \\
\mathrm{PCU}^{\mathrm{a}} \text {, increase in } \\
\text { number of home } \\
\text { deaths, etc. }\end{array}$ & $\begin{array}{l}\text { PC home care improves } \\
\text { continuity in care of patients. } \\
\text { Transfers to intermediate stay } \\
\text { care centers from } 112(14,7 \%) \\
\text { to } 144(21,5 \%)(p=0,001) \text { and } \\
\text { deaths at home increased } \\
\text { from } 61(8 \%) \text { to } 97(14,5 \%) \\
(p=0,000) \text {. Median stay at the } \\
P C U^{a} \text { decreased from } 7 \text { to } \\
6 \text { days }(p=0,155) \text {. }\end{array}$ \\
\hline Navarro et al., 2011.[1 1] & Advanced Chronic Disease & $\begin{array}{l}\text { Observational, } \\
\text { retrospective } \\
\text { and descriptive } \\
\text { study }\end{array}$ & 26 & $\begin{array}{l}\text { EoLC } C^{\text {a }} \text { of advanced } \\
\text { chronic non-cancer } \\
\text { patients identified } \\
\text { by multidimensional } \\
\text { evaluation and interdis- } \\
\text { ciplinary teamwork in a } \\
\text { medium and long term } \\
\text { hospital. }\end{array}$ & $\begin{array}{l}\text { General data, terminal } \\
\text { criteria, diagnostic and } \\
\text { prognostic information, } \\
\text { development of } \\
\text { advance directives, } \\
\text { limiting levels of effort } \\
\text { care, times from } \\
\text { admission, risk of } \\
\text { complicated } \\
\text { bereavement. }\end{array}$ & $\begin{array}{l}\text { Identification of advanced } \\
\text { chronic non-cancer patients } \\
\text { and their needs by interdiscip- } \\
\text { linary teamwork enabled indi- } \\
\text { cation for PC soon after } \\
\text { admission (median } 7 \text { days, } \\
15 \text { days pure palliative treat- } \\
\text { ment) and ensured appropriate } \\
\text { care during their stay (prognostic } \\
\text { to the family, } \\
\text { increased from } 65 \% \text { to } 92 \% \text {; } \\
\text { advance directives from } 25 \% \text { to } \\
96 \% \text {; adequacy level of care ef- } \\
\text { fort increased; Zarit score de- } \\
\text { creased, and risk of } \\
\text { a complicated bereavement, } \\
5 \% \text {. }\end{array}$ \\
\hline
\end{tabular}


Table 6 Clinical guidelines and pathways identified $(n=12)$

\begin{tabular}{|c|c|c|c|c|c|c|c|}
\hline Category & Reference & Date & Title & Disease & Setting & $\begin{array}{l}\text { Emmanuel's criteria }{ }^{a} \\
n(\%)\end{array}$ & Recommendations based on... \\
\hline $\begin{array}{l}\text { Guidelines in } \\
\text { non-cancer }\end{array}$ & Aldasoro et al. [22] & 2012 & $\begin{array}{l}\text { Necesidades en cuidados paliativos } \\
\text { de las enfermedades no oncológicas. } \\
\text { Un estudio cualitativo desde la } \\
\text { perspectiva de profesionales, pacientes } \\
\text { y personas cuidadoras (Needs in PC of } \\
\text { the non oncologic diseases. A qualitative } \\
\text { study from the professionals perspective, } \\
\text { patients and carers) }\end{array}$ & Non cancer & All settings & $4(36 \%)$ & $\begin{array}{l}\text { Other options: Mixed methods } \\
\text { corresponding to the "focused } \\
\text { ethnography" }\end{array}$ \\
\hline $\begin{array}{l}\text { Pathways in } \\
\text { non-cancer }\end{array}$ & Arnedillo et al. [23] & 2012 & $\begin{array}{l}\text { Consenso sobre Atención Integral de las } \\
\text { Agudizaciones de la Enfermedad } \\
\text { Pulmonar Obstructiva Crónica } \\
\text { ATINA-EPOC (Consensus on integrated } \\
\text { care of acute exacerbations of chronic } \\
\text { obstructive pulmonary disease } \\
\text { ATINA-EPOC) }\end{array}$ & COPD & Not applicable & $8(73 \%)$ & Consensus methods only \\
\hline $\begin{array}{l}\text { Pathways in } \\
\text { non-cancer }\end{array}$ & Gómez-Batiste et al. [24] & 2011 & $\begin{array}{l}\text { Proyecto NECPAL CCOMS-ICO. } \\
\text { Identificación y atención integral- } \\
\text { integrada de personas con enfermedades } \\
\text { crónicas avanzadas en servicios de salud } \\
\text { y sociales (NECPAL CCOMS-ICO Project. } \\
\text { Identification and Integral-integrated } \\
\text { attention of patients with advanced } \\
\text { chronic diseases in health and social } \\
\text { services) }\end{array}$ & $\begin{array}{l}\text { Advanced chronic } \\
\text { diseases }\end{array}$ & All settings & $5(45 \%)$ & $\begin{array}{l}\text { Systematic review and consensus } \\
\text { methods }\end{array}$ \\
\hline $\begin{array}{l}\text { Pathway general } \\
\text { approach }\end{array}$ & Agustín et al. [17] & 2011 & $\begin{array}{l}\text { Manual para el manejo del paciente en } \\
\text { Cuidados Paliativos en Urgencias } \\
\text { Extrahospitalarias (Manual for patient } \\
\text { management in PC in Extrahospital } \\
\text { emergencies) }\end{array}$ & Cancer & $\begin{array}{l}\text { Emergencies outside } \\
\text { the hospitals }\end{array}$ & $4(36 \%)$ & Consensus methods only \\
\hline $\begin{array}{l}\text { Guidelines general } \\
\text { approach }\end{array}$ & SECPAL [15] & 2010 & $\begin{array}{l}\text { Guía de Cuidados Paliativos (Palliative } \\
\text { Care Guideline) }\end{array}$ & $\begin{array}{l}\text { Cáncer and non } \\
\text { cáncer }\end{array}$ & All settings & $3(27 \%)$ & Unclear methods \\
\hline $\begin{array}{l}\text { Guidelines general } \\
\text { approach }\end{array}$ & Colomer et al. [16] & 2009 & $\begin{array}{l}\text { Unidad de Cuidados Paliativos: } \\
\text { Estándares y recomendaciones (Palliative } \\
\text { Care Unit: Standards and } \\
\text { recommendations) }\end{array}$ & $\begin{array}{l}\text { Cáncer and non } \\
\text { cáncer }\end{array}$ & All settings & $8(73 \%)$ & $\begin{array}{l}\text { Systematic review and consensus } \\
\text { methods }\end{array}$ \\
\hline $\begin{array}{l}\text { Guidelines general } \\
\text { approach }\end{array}$ & Arrieta et al. [13] & 2008 & $\begin{array}{l}\text { Guía de Práctica Clínica sobre Cuidados } \\
\text { Paliativos (Clinical practical guideline on } \\
\text { Palliative care) }\end{array}$ & $\begin{array}{l}\text { Cancer and non } \\
\text { cancer }\end{array}$ & All settings & $10(91 \%)$ & $\begin{array}{l}\text { Systematic review, consensus } \\
\text { methods, evidence based and } \\
\text { quality assessment }\end{array}$ \\
\hline
\end{tabular}


Table 6 Clinical guidelines and pathways identified $(n=12)$ (Continued)

\begin{tabular}{|c|c|c|c|c|c|c|c|}
\hline $\begin{array}{l}\text { Guidelines general } \\
\text { approach }\end{array}$ & González et al. [14] & 2008 & $\begin{array}{l}\text { Guía de Cuidados Paliativos de la } \\
\text { Comunidad de Madrid (Palliative Care } \\
\text { guidelines of the Autonomous } \\
\text { Community of Madrid) }\end{array}$ & $\begin{array}{l}\text { Cancer and non } \\
\text { cancer }\end{array}$ & All settings & $5(45 \%)$ & Unclear methods \\
\hline $\begin{array}{l}\text { Pathways general } \\
\text { approach }\end{array}$ & Cía et al. [18] & 2007 & $\begin{array}{l}\text { Proceso asistencial integrado de } \\
\text { Cuidados Paliativos (Palliative Care } \\
\text { Integrated assistential Process) }\end{array}$ & $\begin{array}{l}\text { Cancer and non } \\
\text { cancer }\end{array}$ & $\begin{array}{l}\text { Home and hospital } \\
\text { settings }\end{array}$ & 7 (63 \%) & $\begin{array}{l}\text { Systematic review and consensus } \\
\text { methods }\end{array}$ \\
\hline Guidelines in cancer & Carvajal et al. [19] & 2006 & $\begin{array}{l}\text { Guía de recomendaciones clínicas: } \\
\text { Cáncer colorrectal (Clinical } \\
\text { recommendation guideline: Colon } \\
\text { cancer) }\end{array}$ & Colorrectal cancer & All settings & $4(36 \%)$ & $\begin{array}{l}\text { Systematic review and consensus } \\
\text { methods }\end{array}$ \\
\hline Pathways in cancer & Naveira et al. [53] & 2005 & $\begin{array}{l}\text { Cuidados paliativos en el enfermo } \\
\text { oncologico. Documentos para la gestión } \\
\text { integrada de procesos asistenciales } \\
\text { relacionados con el cancer. Proyecto } \\
\text { Oncoguias (Palliative Care for the } \\
\text { oncologic patient. Documents for } \\
\text { integrated management of assitential } \\
\text { processes related to Cancer. "Oncoguías" } \\
\text { Project) }\end{array}$ & Cancer & All settings & $4(36 \%)$ & Unclear methods \\
\hline $\begin{array}{l}\text { Pathways general } \\
\text { approach }\end{array}$ & Hernández et al. [17] & 2004 & $\begin{array}{l}\text { Programa de cuidados domiciliarios en } \\
\text { atención primaria (Home Care program } \\
\text { in Primary attention) }\end{array}$ & $\begin{array}{l}\text { Cancer and non } \\
\text { cancer }\end{array}$ & Home setting & $6(56 \%)$ & Unclear \\
\hline
\end{tabular}

"The 11 aspects assessed by "Emmanuel's" are: discussion of illness limitations and prognosis; recommendations for conducting a whole patient assessment including the patient's physical, social, psychological and spiritual issues, their family and community setting; recommendations for when to make these recommendations; recommendations on when PC should be integrated; assessment of the patient's goals for care, continuous goal adjustment as the illness and the person's disease progresses, PC interventions to reduce suffering, advance care planning, recommendations on involving a PC team, recommendations on PC at the last moments of life and recommendations on grief and bereavement [10]

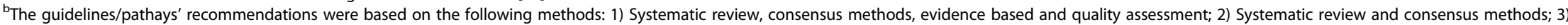
Systematic review only; 4) Consensus methods only; 5) Unclear methods; 6) Other options 
Table 7 Descriptive, strategic and analytical studies on PC integration in Spain $(n=35)$

\begin{tabular}{|c|c|c|c|c|c|c|c|c|}
\hline Category & Reference & Date & Documents titles & Type & Disease & Design & Setting & $\begin{array}{l}\text { Total Hawker } \\
\text { Score(1) }\end{array}$ \\
\hline \multirow[t]{4}{*}{$\begin{array}{l}\text { Descriptive } \\
\text { documents } \\
(n=4)\end{array}$} & $\begin{array}{l}\text { Alberola } \\
\text { et al. [26] }\end{array}$ & 2001 & $\begin{array}{l}\text { Modelos de Cuidados Paliativos en pacientes } \\
\text { con cáncer (Pallitave Care models in } \\
\text { cancer patients) }\end{array}$ & Models & Cancer & $\begin{array}{l}\text { Models } \\
\text { description }\end{array}$ & $\begin{array}{l}\text { General University Hospital } \\
\text { of Valencia; Oncology Catalan } \\
\text { Institute, Barcelona; University } \\
\text { Hospital "Dr Negrín", Las Palmas; } \\
\text { Clinic University Hospital } \\
\text { of Valladolid }\end{array}$ & 10 \\
\hline & $\begin{array}{l}\text { Arrieta } \\
\text { et al. [21] }\end{array}$ & 2012 & $\begin{array}{l}\text { Cuidados paliativos. Proceso asistencial integrado } \\
\text { Araba (Palliative Care. "Araba" Integrated } \\
\text { assistential Process) }\end{array}$ & Models & $\begin{array}{l}\text { Cancer and } \\
\text { non-cancer }\end{array}$ & $\begin{array}{l}\text { Model } \\
\text { description }\end{array}$ & None & $\begin{array}{l}\text { Not } \\
\text { applicable }\end{array}$ \\
\hline & $\begin{array}{l}\text { Pascual N. } \\
{[27]}\end{array}$ & 2011 & $\begin{array}{l}\text { Modelos de atención a pacientes oncológicos } \\
\text { terminales en Andalucía: una mirada sociológica } \\
\text { (Models of oncologic terminal patients attention } \\
\text { in Andalucia) }\end{array}$ & Models & Cancer & $\begin{array}{l}\text { Multimode: } \\
\text { Quantitative and } \\
\text { qualitative tools }\end{array}$ & None & 28 \\
\hline & $\begin{array}{l}\text { Rubi et al. } \\
\text { [25] }\end{array}$ & 2005 & $\begin{array}{l}\text { Cuidados Paliativos en las enfermedades crónicas } \\
\text { en fase avanzada. Situación actual y propuesta de } \\
\text { organización asistencial (Palliative Care in } \\
\text { advanced chronic respiratory disease. Status and } \\
\text { assistential proposal for organisation) }\end{array}$ & $\begin{array}{l}\text { Review and } \\
\text { proposal }\end{array}$ & $\begin{array}{l}\text { Advanced } \\
\text { chronic } \\
\text { respiratory } \\
\text { disease }\end{array}$ & Narrative review & None & 16 \\
\hline \multirow[t]{4}{*}{$\begin{array}{l}\text { Strategic } \\
\text { documents } \\
(n=17)\end{array}$} & $\begin{array}{l}\text { Gómez-Batiste } \\
\text { et al. [37] }\end{array}$ & 2013 & $\begin{array}{l}\text { Identificación de personas con enfermedades } \\
\text { crónicas avanzadas y neceisdad de cuidados } \\
\text { paliativos en los servicios socio-sanitarios: } \\
\text { herramienta NECPAL CCOMS-ICO@ (Identification } \\
\text { of people with chronic advanced diseases and } \\
\text { need of palliative care in sociosanitary services: } \\
\text { elaboration of the NECPAL CCOMS-ICO@ tool) }\end{array}$ & $\begin{array}{l}\text { Concrete } \\
\text { situations }\end{array}$ & $\begin{array}{l}\text { Advanced } \\
\text { chronic } \\
\text { diseases }\end{array}$ & Descriptive & None & 15 \\
\hline & Miguez C. [33] & 2010 & $\begin{array}{l}\text { Guía formativa del residente de oncología } \\
\text { radioterápica. (Training guideline for resident of } \\
\text { radiotherapic oncology) }\end{array}$ & $\begin{array}{l}\text { Concrete } \\
\text { situations }\end{array}$ & $\begin{array}{l}\text { Radiotherapic } \\
\text { oncology }\end{array}$ & Not applicable & None & $\begin{array}{l}\text { Not } \\
\text { applicable }\end{array}$ \\
\hline & $\begin{array}{l}\text { Castellanos } \\
\text { et al. [35] }\end{array}$ & 2007 & $\begin{array}{l}\text { Plan integral de atención sociosanitaria al } \\
\text { deterioro cognitivo en Extremadura PIDEX } \\
\text { (Integral plan of sociosanitary attention to } \\
\text { cognitive impairment in Extremadura PIDEX) }\end{array}$ & $\begin{array}{l}\text { Concrete } \\
\text { situations }\end{array}$ & $\begin{array}{l}\text { Cognitive } \\
\text { impairment }\end{array}$ & Not applicable & None & $\begin{array}{l}\text { Not } \\
\text { applicable }\end{array}$ \\
\hline & $\begin{array}{l}\text { Herrera et al. } \\
{[54]}\end{array}$ & 2006 & $\begin{array}{l}\text { Primer nivel asistencial en Cuidados paliativos: } \\
\text { Evolución del contenido de la cartera de servicios } \\
\text { de atención primaria y criterios de derivación al } \\
\text { nivel de soporte.(Primary Palliative Care: } \\
\text { Development of the contents of the primary care } \\
\text { services portfolio and criteria for referral according } \\
\text { to complexity) }\end{array}$ & $\begin{array}{l}\text { Concrete } \\
\text { situations }\end{array}$ & $\begin{array}{l}\text { Cancer and } \\
\text { non-cancer }\end{array}$ & Not applicable & None & 19 \\
\hline
\end{tabular}


Table 7 Descriptive, strategic and analytical studies on PC integration in Spain $(n=35)$ (Continued)

\begin{tabular}{|c|c|c|c|c|c|c|c|}
\hline $\begin{array}{l}\text { Gómez-Batiste } \\
\text { et al. [45] }\end{array}$ & 2012 & $\begin{array}{l}\text { Proyecto de demonstración de la Organización } \\
\text { Mundial de la Salud de Cataluña sobre } \\
\text { implementación de cuidados paliativos: resultados } \\
\text { cuantitativos y cualitativos en } 20 \text { años (The Catalonia } \\
\text { World Health Organization demonstration project for } \\
\text { palliative care implementation: quantitative } \\
\text { and } \\
\text { qualitative results at } 20 \text { years) }\end{array}$ & Assessment & $\begin{array}{l}\text { Cancer and } \\
\text { non-cancer } \\
\text { diseases }\end{array}$ & $\begin{array}{l}\text { Quantitative and } \\
\text { qualitative }\end{array}$ & None & 20 \\
\hline $\begin{array}{l}\text { Gómez-Batiste } \\
\text { et al. }\end{array}$ & 2007 & $\begin{array}{l}\text { Proyecto de demonstración de la Organización } \\
\text { Mundial de la Salud de Cataluña en } 15 \text { años } \\
\text { (Catalonia WHO palliative care demonstration } \\
\text { project at } 15 \text { Years. 2005) }\end{array}$ & Assessment & $\begin{array}{l}\text { Cancer and } \\
\text { non-cancer }\end{array}$ & Unclear & None & 20 \\
\hline $\begin{array}{l}\text { Gómez-Batiste } \\
\text { et al. [39] }\end{array}$ & 2006 & $\begin{array}{l}\text { Consumo de recursos y costes de los servicios de } \\
\text { cuidados paliativos en España: un studio } \\
\text { prospective multi-céntrico. (Resource consumption } \\
\text { and costs of palliative care services in Spain: a } \\
\text { multicenter prospective study) }\end{array}$ & Assessment & $\begin{array}{l}\text { Cancer and } \\
\text { non-cancer }\end{array}$ & $\begin{array}{l}\text { Descriptive- } \\
\text { observational, } \\
\text { prospective, } \\
\text { longitudinal } \\
\text { Multicenter } \\
\text { Study }\end{array}$ & None & 22 \\
\hline Cía et al. [28] & 2008 & $\begin{array}{l}\text { Plan andaluz de Cuidados Paliativos (Andalusian } \\
\text { plan for palliative care) }\end{array}$ & Regional Plan & $\begin{array}{l}\text { Cancer and } \\
\text { non-cancer }\end{array}$ & Not applicable & None & $\begin{array}{l}\text { Not } \\
\text { applicable }\end{array}$ \\
\hline $\begin{array}{l}\text { Gago et al. } \\
{[29]}\end{array}$ & 2009 & $\begin{array}{l}\text { Estrategia de Cuidados Paliativos para Asturias } \\
\text { (Palliative care strategy for Asturias) }\end{array}$ & Regional Plan & $\begin{array}{l}\text { Cancer and } \\
\text { non-cancer }\end{array}$ & Not applicable & None & $\begin{array}{l}\text { Not } \\
\text { applicable }\end{array}$ \\
\hline $\begin{array}{l}\text { Amorín et al. } \\
{[30]}\end{array}$ & 2009 & $\begin{array}{l}\text { Programa de Cuidados Paliativos de Aragón } \\
\text { (Palliative care strategy for Aragón) }\end{array}$ & Regional Plan & $\begin{array}{l}\text { Cancer and } \\
\text { non-cancer }\end{array}$ & Not applicable & None & $\begin{array}{l}\text { Not } \\
\text { applicable }\end{array}$ \\
\hline $\begin{array}{l}\text { López et al. } \\
\text { [31] }\end{array}$ & 2010 & $\begin{array}{l}\text { Plan integral de Cuidados paliativos de la } \\
\text { comunidad Valenciana (Palliative care integral } \\
\text { plan for the Comunidad Valenciana) }\end{array}$ & Regional Plan & $\begin{array}{l}\text { Cáncer and } \\
\text { non-cancer }\end{array}$ & Not applicable & None & $\begin{array}{l}\text { Not } \\
\text { applicable }\end{array}$ \\
\hline Unknown & Unknown & $\begin{array}{l}\text { Programa Integral Atención Paliativa Cantabria } \\
\text { (Palliative integral attention programme } \\
\text { for Cantabria) }\end{array}$ & Regional Plan & $\begin{array}{l}\text { Cáncer and } \\
\text { non-cancer }\end{array}$ & Not applicable & None & $\begin{array}{l}\text { Not } \\
\text { applicable }\end{array}$ \\
\hline $\begin{array}{l}\text { Aguilera et al. } \\
\text { [55] }\end{array}$ & 2005 & $\begin{array}{l}\text { Plan integral de Cuidados Paliativos de la } \\
\text { Comunidad de Madrid. 2005-2008 } \\
\text { (Palliative Care integral plan for the } \\
\text { Community of Madrid. } \\
\text { 2005-2008) }\end{array}$ & Regional Plan & $\begin{array}{l}\text { Cáncer and } \\
\text { non-cancer }\end{array}$ & Not applicable & None & $\begin{array}{l}\text { Not } \\
\text { applicable }\end{array}$ \\
\hline $\begin{array}{l}\text { Fernández } \\
\text { et al. [33] }\end{array}$ & 2007 & $\begin{array}{l}\text { Plan Integral de Cuidados Paliativos de la } \\
\text { Comunidad Autónoma de la Región } \\
\text { de Murcia 2006-2009 (Palliative Care integral } \\
\text { plan for the Community of Murcia. 2006-2009) }\end{array}$ & Regional Plan & $\begin{array}{l}\text { Cáncer and } \\
\text { non-cancer }\end{array}$ & Not applicable & None & $\begin{array}{l}\text { Not } \\
\text { applicable }\end{array}$ \\
\hline $\begin{array}{l}\text { García- } \\
\text { Baquero et al. } \\
{[14]}\end{array}$ & 2010 & $\begin{array}{l}\text { Borrador del Plan estratégico de Cuidados } \\
\text { paliativos de la Comunidad de Madrid, } \\
\text { 2010-2014 (Draft of the Palliative Care strategic }\end{array}$ & Regional Plan & $\begin{array}{l}\text { Cáncer and } \\
\text { non-cancer }\end{array}$ & Not applicable & None & $\begin{array}{l}\text { Not } \\
\text { applicable }\end{array}$ \\
\hline
\end{tabular}


Table 7 Descriptive, strategic and analytical studies on PC integration in Spain $(n=35)$ (Continued)

\begin{tabular}{|c|c|c|c|c|c|c|c|}
\hline $\begin{array}{l}\text { Gobierno } \\
\text { vasco [56] }\end{array}$ & 2006 & $\begin{array}{l}\text { Plan de atención a pacientes en la fase final de } \\
\text { la vida o Cuidados Paliativos de la Comunidad } \\
\text { autónoma vasca (Plan of patients attention in } \\
\text { end of life stage or Palliative Care in the Basque } \\
\text { Autonomous región) }\end{array}$ & Regional Plan & $\begin{array}{l}\text { Cancer and } \\
\text { non-cancer }\end{array}$ & Not applicable & None & $\begin{array}{l}\text { Not } \\
\text { applicable }\end{array}$ \\
\hline $\begin{array}{l}\text { Pascual et al. } \\
\text { [2] }\end{array}$ & 2011 & $\begin{array}{l}\text { Estrategia en Cuidados Paliativos del Sistema } \\
\text { Nacional de Salud. Actualización 2010-2014 } \\
\text { (Palliative Care Strategy of the National Health } \\
\text { Service. Update 2010-2014) }\end{array}$ & National Plan & $\begin{array}{l}\text { Cancer and } \\
\text { non-cancer }\end{array}$ & Not applicable & None & $\begin{array}{l}\text { Not } \\
\text { applicable }\end{array}$ \\
\hline $\begin{array}{l}\text { Rocafort et al. } \\
{[57]}\end{array}$ & 2006 & $\begin{array}{l}\text { Equipos de soporte de cuidados paliativos y } \\
\text { dedicación de los equipos de atención } \\
\text { primaria a pacientes en situación terminal } \\
\text { en sus domicilios (Palliative care support } \\
\text { teams and the commitment of primary } \\
\text { care teams to terminally ill patients } \\
\text { in their homes) }\end{array}$ & Observational & $\begin{array}{l}\text { Cancer and } \\
\text { non-cancer }\end{array}$ & $\begin{array}{l}\text { Multicentre } \\
\text { observational } \\
\text { study. }\end{array}$ & None & 19 \\
\hline $\begin{array}{l}\text { Ko W et al. } \\
{[58]}\end{array}$ & 2013 & $\begin{array}{l}\text { Awareness of general practitioners concerning } \\
\text { cancer patients' preferences for place of death: } \\
\text { evidence from four European countries. }\end{array}$ & Observational & Cancer & $\begin{array}{l}\text { Retrospective } \\
\text { study }\end{array}$ & None & 28 \\
\hline $\begin{array}{l}\text { Alonso et al. } \\
\text { [59] }\end{array}$ & 1997 & $\begin{array}{l}\text { Atención al paciente oncológico terminal } \\
\text { en un distrito de atención primaria } \\
\text { (Care for the terminal cancer patient } \\
\text { in a primary care district) }\end{array}$ & Observational & Cancer & Unkown & None & $\begin{array}{l}\text { Not } \\
\text { applicable }\end{array}$ \\
\hline $\begin{array}{l}\text { Canal et al. } \\
{[60]}\end{array}$ & 2005 & $\begin{array}{l}\text { Valoración de la implantación de un } \\
\text { Programa Integral de Cuidados Paliativos. } \\
\text { Visión retrospectiva 1995-1999 (Evaluation of } \\
\text { the developement of a palliative car program. } \\
\text { Review from } 1995 \text { to 1999) }\end{array}$ & Observational & Cancer & $\begin{array}{l}\text { Retrospective } \\
\text { and descriptive } \\
\text { included in the } \\
\text { ecologic } \\
\text { category, by } \\
\text { revising clinical } \\
\text { records }\end{array}$ & $\begin{array}{l}\text { Home care, inpatients units in } \\
\text { long term and Inpatient Units } \\
\text { in General Hospitals }\end{array}$ & 18 \\
\hline $\begin{array}{l}\text { Simó et al. } \\
\text { [61] }\end{array}$ & 2006 & $\begin{array}{l}\text { Seguimiento de los pacientes atendidos } \\
\text { conjuntamente por un equipo de Atención } \\
\text { Primaria y su programa de Atención } \\
\text { Domiciliaria y Equipos de Soporte } \\
\text { (Follow-up of patients jointly cared for by a } \\
\text { Primary Care unit, its Home Care program, } \\
\text { and a Home Care Support Unit) }\end{array}$ & Observational & $\begin{array}{l}\text { Multiple } \\
\text { patologies: } \\
\text { Dementia, } \\
\text { neumologic } \\
\text { diseases, } \\
\text { neurologic } \\
\text { diseases, } \\
\text { cardiologic } \\
\text { diseases, } \\
\text { cancer, } \\
\text { etcetera.. }\end{array}$ & $\begin{array}{l}\text { Descriptive and } \\
\text { retrospective } \\
\text { study }\end{array}$ & $\begin{array}{l}\text { Urban basic health area of } \\
\text { Terrasa (Barcelona) }\end{array}$ & 21 \\
\hline $\begin{array}{l}\text { Riera et al. } \\
{[62]}\end{array}$ & 2008 & $\begin{array}{l}\text { Resultados de la evaluación de un } \\
\text { instrumento de trabajo interdisciplinar: } \\
\text { trayectoria clínica de la Unidad de } \\
\text { Cuidados Paliativos (Implementation of }\end{array}$ & Observational & $\begin{array}{l}\text { Advanced or } \\
\text { terminal } \\
\text { cáncer }\end{array}$ & $\begin{array}{l}\text { A retrospective } \\
\text { study of clinical } \\
\text { records }\end{array}$ & $\begin{array}{l}\text { "Hospital de la Esperanza", } \\
\text { Barcelona, Spain. }\end{array}$ & 22 \\
\hline
\end{tabular}

Analytical studies Rocafort et al. 2006 $(n=14)$ trayectoria clínica de la Unidad de an interdisciplinary working tool: Palliative Care Unit clinical pathway. Results of its evaluation: 
Table 7 Descriptive, strategic and analytical studies on PC integration in Spain $(n=35)$ (Continued)

\begin{tabular}{|c|c|c|c|c|c|c|c|}
\hline $\begin{array}{l}\text { Costa et al. } \\
{[63]}\end{array}$ & 2012 & $\begin{array}{l}\text { Demencia avanzada y cuidados paliativos, } \\
\text { características sociodemográficas y clínicas } \\
\text { (Advanced dementia and palliative care, } \\
\text { socio-demographic and clinical characteristics) }\end{array}$ & Observational & $\begin{array}{l}\text { Advanced } \\
\text { dementia }\end{array}$ & $\begin{array}{l}\text { Observational } \\
\text { descriptive }\end{array}$ & $\begin{array}{l}\text { Antic Hospital St. Jaume i Sta } \\
\text { Magdalena (Mataró, Barcelona) }\end{array}$ & 22 \\
\hline $\begin{array}{l}\text { De Santiago } \\
\text { et al. [64] }\end{array}$ & 2012 & $\begin{array}{l}\text { Un nuevo equipo de soporte hospitalario en el } \\
\text { departamento de oncología de un hospital } \\
\text { universitario: evaluación de eficacia inicial y eficiencia. } \\
\text { (A new palliative care consultation team at the } \\
\text { oncology department of a university hospital: an } \\
\text { assessment of initial efficiency and effectiveness) }\end{array}$ & Experimental & $\begin{array}{l}\text { Cancer and } \\
\text { non-cancer }\end{array}$ & $\begin{array}{l}\text { Retrospective } \\
\text { study }\end{array}$ & $\begin{array}{l}\text { University of Navarre Clinic, } \\
\text { Oncology department. } \\
\text { Pamplona, Spain }\end{array}$ & 22 \\
\hline $\begin{array}{l}\text { Alonso- } \\
\text { Babarro et al. } \\
\text { [65] }\end{array}$ & 2013 & $\begin{array}{l}\text { La asociación entre la muerte del paciente, } \\
\text { utilzación de recursos hospitalarios y disponibilidad } \\
\text { de servicios domiciliarios para pacientes con cáncer. } \\
\text { (The association between in-patient death, utilization } \\
\text { of hospital resources and availability of palliative } \\
\text { homecare for cancer patients) }\end{array}$ & Experimental & Cancer & $\begin{array}{l}\text { Population-based } \\
\text { study }\end{array}$ & $\begin{array}{l}\text { Alcobendas-San-Sebastian de } \\
\text { Los Reyes and Alcala de } \\
\text { Henares districts }\end{array}$ & 31 \\
\hline $\begin{array}{l}\text { Vega et al. } \\
{[66]}\end{array}$ & 2011 & $\begin{array}{l}\text { Atención sanitaria paliativa y de soporte de los } \\
\text { equipos de atención primaria en el domicilio } \\
\text { (Palliative and support care at home in primary care) }\end{array}$ & Experimental & $\begin{array}{l}\text { Cancer and } \\
\text { non-cancer }\end{array}$ & Descriptive & $\begin{array}{l}\text { Five spanish sentinel networks } \\
\text { between October } 2007 \text { and } \\
\text { march 2008, in five } \\
\text { Autonomous regions: } \\
\text { Comunidad Valenciana, La Rioja, } \\
\text { Castilla y León, Asturias and } \\
\text { Extremadura }\end{array}$ & 16 \\
\hline $\begin{array}{l}\text { Prades et al. } \\
\text { [67] }\end{array}$ & 2011 & $\begin{array}{l}\text { Tratamiento multidisciplinar en cancer en España, } \\
\text { o cuando la función crea el órgano: estudio de } \\
\text { entrevista cualitativa. (Multidisciplinary cancer } \\
\text { care in Spain, or when the function creates the } \\
\text { organ: qualitative interview study) }\end{array}$ & Experimental & Cancer & $\begin{array}{l}\text { Qualitative } \\
\text { interview study } \\
\text { with semi- } \\
\text { structured, one- } \\
\text { to-one interviews }\end{array}$ & $\begin{array}{l}\text { Most populated regions of } \\
\text { Spain, namely, Andalusia, } \\
\text { Catalonia, Madrid, Galicia and } \\
\text { Valencia }\end{array}$ & 19 \\
\hline $\begin{array}{l}\text { Colchero } \\
\text { et al. [68] }\end{array}$ & 2009 & $\begin{array}{l}\text { Atención en pacientes oncológicos terminals } \\
\text { en un distrito de atención primaria (Care of } \\
\text { terminally ill oncology patients in an urban } \\
\text { primary care district) }\end{array}$ & Experimental & Cancer & $\begin{array}{l}\text { Transversal } \\
\text { descriptive study }\end{array}$ & $\begin{array}{l}2 \text { hospital areas in the Seville's } \\
\text { primary attention district, } 32 \\
\text { centres and } 278 \text { patients }\end{array}$ & 11 \\
\hline $\begin{array}{l}\text { Agra et al. } \\
\text { [69] }\end{array}$ & 2003 & $\begin{array}{l}\text { Relación de la calidad de vida con diferentes } \\
\text { modelos de atención domiciliaria en enfermos } \\
\text { oncológicos terminales de un área sanitaria } \\
\text { de Madrid (Relationship between quality of life }\end{array}$ & Experimental & Cancer & $\begin{array}{l}\text { A quasi- } \\
\text { experimental } \\
\text { prospective } \\
\text { study }\end{array}$ & $\begin{array}{l}\text { Area } 4 \text { of the "Imsalud" in } \\
\text { Madrid }\end{array}$ & 25 \\
\hline
\end{tabular}

oncology patients from a health area of Madrid)

study 
Table 7 Descriptive, strategic and analytical studies on PC integration in Spain ( $n=35)$ (Continued)

\begin{tabular}{|c|c|c|c|c|c|c|c|}
\hline $\begin{array}{l}\text { Rihuete et al. } \\
\text { [70] }\end{array}$ & 2005 & $\begin{array}{l}\text { Atención integral al paciente oncológico y su familia } \\
\text { desde una intervención multidisciplinar (Integral } \\
\text { attention to oncology patients and their relatives } \\
\text { from a multidisciplinary team) }\end{array}$ & Experimental & Cancer & $\begin{array}{l}\text { Retrospective } \\
\text { analysis of social } \\
\text { interventions } \\
\text { and a new } \\
\text { methodology of } \\
\text { proactive } \\
\text { intervention, } \\
\text { employing } \\
\text { multidisciplinary } \\
\text { clinic social } \\
\text { sessions. And } \\
\text { finally both } \\
\text { interventions } \\
\text { were compared }\end{array}$ & $\begin{array}{l}\text { Unit of Oncology in the } \\
\text { University of Salamanca }\end{array}$ & 14 \\
\hline
\end{tabular}

(1) Protocol of the Hawker et al. appraisal tool [9] punctuates from 1 (very poor) to 4 (good) the following aspects: 1. Abstract and title: Did they provide a clear description of the study?; 2. Introduction and aims: Was there a good background and clear statement of the aims of the research?; 3. Method and data: Is the method appropriate and clearly explained?; 4 . Sampling: Was the sampling strategy appropriate to address the aims?; 5. Data analysis: Was the description of the data analysis sufficiently rigorous?; 6. Ethics and bias: Have ethical issues been addressed, and what has necessary ethical approval gained? Has the relationship between researchers and participants been adequately considered?; 7. Results: Is there a clear statement of the findings?; 8 . Transferability or generalizability: Are the findings of this study transferable (generalizable) to a wider population?; 9. Implications and usefulness: How important are these findings to policy and practice? 
addressed cancer patients. Four out of 11 Emmanuel's criteria [10] appeared for both and were applicable to all settings. The "Guía de recomendaciones clínicas: cancer colorectal" (Guideline of clinical recommendations: colon cancer) [20] used systematic review and consensus methods, so was considered good quality. Commonly, these two documents include recommendations for conducting a whole patient assessment including patient's physical, social, psychological, and spiritual issues within their family and community setting, and recommendations on grief and bereavement care (Table 6).

\section{Guidelines and pathways on non-cancer}

One clinical guideline [22] and two pathways (one relating to Chronic Obstructive Pulmonary Disease (COPD) and the other, general chronic advanced illness) were identified [23, 24]. They were published between 2011-2012 and apply to all settings. These three rated well in terms of IPC against Emmanuel's criteria [10] (see Table 6). The two pathways share recommendations for conducting a whole patient assessment, including their family and community setting; assessment of the patient's goals of care; continuous goal adjustment as the disease progresses; and the presence of the advance care planning criterion (Table 6).

\section{Analytical comparison of all guidelines and pathways}

Five of the documents ( $42 \%)$ were above the average in Emmanuel's criteria [10] and seven (58 \%) below. All exceeded the initial filter of achieving at least two out of the 11 criteria.

With regard to the recommendations appearing, there exist large variations. The most reported recommendations are: conduction of a whole patient assessment including patient's physical, social, psychological, and spiritual issues in the context of their family and their community setting; recommendations on when PC should be integrated; and suggestions to intervene to reduce suffering as needed. These recommendations were found in three quarters of the selected documents. Conversely, the least cited recommendations are those related to the timing of assessments and to continuous goal adjustment as illness progresses. (Additional file 1: Table S1).

\section{Descriptive, strategic and analytical documents}

Strategic documents $(n=17)$, descriptive documents $(n=4)$, and analytical studies $(n=14)$ were found (Table 7).

\section{Descriptive documents}

Amongst these, there are papers describing diverse programmes with fruitful collaborations between PC teams and oncology departments in a narrative review of patients suffering from advanced chronic respiratory disease [25]; descriptions of an integrated PC process [21, 26] and a description of how care provision for terminal cancer patients was organised in Andalucia in the year 2000 [27].

\section{Strategic documents}

Nine of the strategic documents (53\%) are produced at a regional level (Andalucía [28], Asturias [29], Aragón [30], Comunidad Valenciana [31], Cantabria, Madrid [14, 32], Murcia [33] and the Basque Country [34]), and one (5\%) at a National level. This latter is an update (2010-2014) for the Strategy in Palliative Care of the National Health System released by the Health, Social Policies and Equality Ministry [2].

These strategic documents are particularly important in Spain due to the nature of the Spanish National Health System, as it is a decentralised governmental system where healthcare regions have considerable control on the delivery of health care.

Four other documents (24\%) address integration of PC in concrete situations; radiotherapy [35], cognitive impairment [36], advanced chronic disease [37] and primary care [38].

Finally, a set of three documents (18\%) have been categorized as assessment documents; two of these evaluated resource consumption and cost effectiveness [39, 40], and the other, assessment of PC implementation by a WHO demonstration project [41].

\section{Analytical studies}

These documents were identified within PUBMED $(n=8$, $57 \%$ ) or the Spanish journal Medicina Paliativa (Palliative Medicine) $(n=6,43 \%)$ and included 7 observational and 7 experimental, intervention studies.

Analytical studies mainly focused on integration of PC into primary care, resource utilisation, focusing on cancer, dementia, and other non-cancer conditions. In contrast to the models (noted above), these scored moderately against the Hawker et al. tool, scoring under 22 out of a possible 36 .

The observational studies include descriptive and retrospective designs describing and analysing clinical records, whereas the experimental studies involved proactively engaging with patients and PC professionals as study participants. These adopted diverse study designs including: retrospective, population-based, descriptive, structured and semi-structured interviews, quasiexperimental prospective and prospective cohort studies.

The majority of these addressed cancer $(n=9,64 \%)$, a few refer to cancer and non-cancer diseases $(n=4$, $29 \%$ ), and just one (7\%) considered non-cancer alone. The settings to which these studies apply varied from PC units in long term and general hospitals, home care situations and wider health regions. All these studies conclude that IPC have positive impacts on the quality 
of life of patients and their families, improves patient perception of their own health condition, and reduces inpatient deaths and hospitalizations in the last months of life.

\section{Discussion}

A total of 49 documents including models, clinical guidelines and pathways, and other strategic, descriptive and analytical documents have been identified. The majority of guidelines and pathways scored well against Emmanuel's criteria [10] in terms of PC integration. Strategic, analytical and descriptive studies evaluated with Hawker et al. tool [9] show that for 15 of these (43\%), the score was above half of the total attainable points.

Spain has included PC into guidelines and pathways on cancer in a good theoretical level of integrated PC (as assessed by Emmanuel's criteria) meaning that key point elements for conducting IPC for cancer patients are included within guidelines and pathways, at the time that quality was considered high.

In contrast, the number identified for chronic advanced diseases suggest that Spain is at an early stage if we take chronic obstructive pulmonary disease or chronic heart failure (CHF) as exemplars. Only a few of these could be considered robust in terms of developmental methods and level of evidence, as they are supported mainly on consensus processes, and further evaluation should be applied to evaluate quality. Just one guideline: "Guía de Práctica Clínica sobre Cuidados Paliativos" (Clinical Practice Guideline on Palliative Care) can be considered of high quality matching 10 out of 11 criteria on the Emmanuel scale [10].

Another two documents, the guideline "Unidad de Cuidados Paliativos: Estándares y recomendaciones" (Palliative Care Unit: standards and recommendations) [16] and the pathway "Consenso sobre Atención Integral de las Agudizaciones de la Enfermedad Pulmonar Obstructiva Crónica ATINA-EPOC" (Consensus on integrated care of acute exacerbations of chronic obstructive pulmonary disease ATINA-EPOC) [23], are good examples as contain 8 out of 11 Emmanuel's key recommendations. These -jointly with the previously indicated guideline-, place an emphasis in organisational and clinical aspects bearing in mind the importance of coordination, dialogue and constant relationship between units and assistance levels. All aiming to guarantee continuity of care between all agents involved in the PC process.

Overall, this review demonstrates some progress in IPC by Spanish health providers and policy makers and suggests general agreement on the need for the integration of palliative care in service provision. This is supported by the existence of strategic documents and it seems that planning for IPC is a major concern for the
Spanish Public Health System and its different regional services. That said, implementation plans should be developed beyond the theory $[42,43]$.

The National Health System in Spain included the enhancement of attention to $\mathrm{PC}$ as one of the strategic priorities within the Quality Plan of the National Health System [44]. On this basis, the challenge of integrating multidisciplinary PC teams, attention to continuity of care, and coordination between different levels is already being addressed [3].

Other studies are proceeding similarly, for example by reviewing the current situation of IPC in the region of Catalonia and moreover, identifying possible areas of improvement $[40,45]$. The focus of this paper is Spain, as a whole, and suggests the need for further research on the topic, in order to improve the quality of life and palliative care provision for patients and their families.

International studies have recently investigated IPC at an European level, seeking and analysing guidelines and pathways for adult cancer patients [46], for COPD or CHF patients [47], and another non-published paper seeking empirically-tested models both in cancer and chronic diseases [48]. Similarly to our study, both in cancer and non-cancer guidelines and pathways, most frequent key components of IPC (according to Emmanuel's list) are: holistic approach and suggestions to intervene to reduce suffering as needed [46, 47]. Interestingly, it is noticeable the frequency difference of grief and bereavement contents in guidelines and pathways for noncancer patients between Spain (60 \%) and European countries $(21 \%)$ [47].

Lately, a full and varied body of research has been published including several reviews on IPC from diverse perspectives. Amongst them, there is a review of evidence reporting the positive impact of engaging communities in end-of-life care [49]. Secondly, an integrative review addresses paediatric $\mathrm{PC}$ and psychosocial support in oncology settings, revealing a set of issues to develop comprehensive psychosocial PC standards [50]. A study on barriers/opportunities to IPC in the United States from a public health perspective highlights the lack of education/training, inadequate size of trained workforce and several policy barriers such as regulatory barriers, lack of funding for research, problems in reimbursement and a fragmented healthcare system [51]. Finally, a narrative synthesis reviewing themes that facilitate and hinder collaboration between hospital-based generalist PC professionals and in-patient specialist PC professionals, finds out five themes essential to either enhancing or worsening effective collaboration: model of care, professional onus, expertise and trust, skill building and specialist PC operations [52]. 
Readers should be aware that the number of sources used in this review is limited (PUBMED, Cochrane Library, Cinahl, Google, Medicina Paliativa) although we have considered the most appropriate available for our context. PUBMED includes all the most important journals, Cinahl is the most important nursing database, Medicina Paliativa is the only PC Spanish journal, Cochrane Library was included to explore secondary information and Google brings Scielo's articles as well as grey literature. It must be also acknowledged that the Google hits considered stopped when reaching 800 hits as Google itself orders and allocates in first positions most relevant documents, which does not mean that other results could have been considered.

The concept of IPC itself remains a developing concept with all its implications in terms of robust, published research. This is a first work in this area relating to Spanish-speaking countries. We suggest that others could benefit from this and may seek to replicate our methods to investigate the situation of IPC in their own countries literature.

A beneficial approach for the future might be to test whether models, plans, guidelines and pathways, as outlined above, have been used with positive effect and demonstrable service improvements for patients and families in receipt of palliative care. These aspects could allow to compare and implement a kind of benchmarking which could be useful for policy makers and managers among others.

\section{Conclusions}

The existence of scarce implemented IPC models, the number and quality of clinical guidelines and pathways, and the large amount of other relevant documents addressing IPC seem to demonstrate that IPC is at an incipient development stage in Spain.

Documents from a strategic, descriptive and analytical perspective, overall point out the achievements in terms of policy makers and health providers agreements and contextualise a potential environment.

From our review, it can be said that first steps towards IPC in Spain have been made, but the literature lacks sufficient evidence about implementation and therefore highlights that much work remains to be done.

\section{Ethics approval and consent to participate}

There are no human participants involved.

\section{Consent for publication}

There are no any individual person's data.

\section{Availability of data and materials}

Not applicable.

\section{Additional file}

Additional file 1: Display of the Emmanuel's criteria per study. (DOCX $25 \mathrm{~kb}$ )

\section{Abbreviations}

CHF: chronic heart failure; COPD: chronic obstructive pulmonary disease; IPC: integrated palliative care; PC: palliative care; WHO: World Health Organisation.

\section{Competing interests}

The authors declare that they have no competing interest.

\section{Authors' contributions}

EG has contributed to the study design, collected data, analysed the information and wrote the paper; $\mathrm{JH}$ has carried out the conceptual framework for the study, contributed to the study design, drafted and revised critically the paper and given final approval of the version; JMC has contributed to the study design and supported methodologically the study in data collection and analysis, he has revised the draft papers and made intellectual contributions; KVB has carried out the study design, defined the protocol, supported with the analysis and given feedback in draft papers author's rounds before submission; NS has carried out the study design, defined the protocol, supported with the analysis and given feedback in draft papers author's rounds before submission; AC has participated in the study design, in protocol's agreement, in data collection and of the draft papers prior to submission; JM has supervised the study design, protocol, and critically and intellectually contributed to the draft versions of the study prior to submission; CC has contributed to the study design, plus refined the study protocol, collected data, supervised the analysis, critically and intellectually revised the paper and approved the final version for submission. All authors read and approved the final manuscript.

\section{Authors' information}

EG, history degree background, specialist in history archives and research assistant at the Palliative Care Atlantes Research Programme, University of Navarra (Spain)

$\mathrm{JH}$, assistant Professor in Radboud University Medical Centre Nijmegen, researcher and project leader and coordinator of the InSup-C EU- on integrated Palliative Care as an European FP7 funded project

JMC, graduate in Political Sciences and Sociology, and doctor in Public Health, experienced researcher in epidemiology (population and clinic) and in public health fields, using both quantitative, qualitative and consensus methodologies. Researcher at the ATLANTES Programme, University of Navarra. KVB, oncologist at the Dept. of Radiation-Oncology and Palliative Medicine, University Hospital Gasthuisberg, Leuven, Belgium; and researcher involved in the InSup-C European project on integrated palliative care.

NS, psychologist who is receiving doctoral training in Biomedical Sciences (Leuven, Belgium) and researcher involved in the InSup-C European project on integrated palliative care.

AC, Medical Director at the Pecs-Baranya Hospice Foundation, provides patient care and palliative care consults for primary care physicians. She is an Associate Professor at the Institute of Family Medicine, University of Pecs Medical School, Hungary teaching medical, nursing and pharmacy students, residents and continuing medical education courses.

$J M$, radiation Oncologist and coordinator palliative care for the palliative support team and the palliative care unit of 12 beds in the University Hospital, Leuven. Dr. Menten is an Active collaborator in FP-7 EU projects Europall, Prisma and Impact and has special clinical interest in cancer pain management.

CC, consultant in Palliative Medicine at the University of Navarra Clinic. Dr Centeno leads a research programme called Atlantes, at the Institute for Culture and Society (ICS), University of Navarra, applying the approach of Humanities and Social Sciences to advanced diseases and palliative care. Besides, teaches at the undergraduate level at the University of Navarra and works in several EAPC Task Forces.

\section{Acknowledgements}

This systematic review is an adaptation of an ongoing European project (InSup-C: www.insup-c.eu) on IPC in six countries (Germany, United 
Kingdom, Belgium, Hungary, Netherlands and Spain). The Spanish team has followed the same aim and adapted the method, and continues other studies on the topic already investigating if PC is integrated or not into the Spanish National Health System, at different assistance levels, and into non-cancer conditions

InSup-C, patient-centered integrated palliative care pathways in advanced cancer and chronic disease, is funded by the European Union Seventh Framework Programme (FP7/HEALTH, under grant agreement 305555). InSup-C aims to identify the prerequisites for best practice in integrated palliative care with the overall objective of improving service delivery and service user outcomes. InSup-C is coordinated by Dr Jeroen Hasselaar, BSC, PhD of Radboud University Medical Center, Nijmegen, The Netherlands. Other partners are: University Hospital, Bonn, Germany; International Observatory on End-of-Life Care, Lancaster University, Lancaster, United Kingdom; University of Pecs Medical School, Pecs, Hungary; University Hospital Leuven, Leuven, Belgium; World Health Organization, Geneva, Switzerland; European Association for Palliative Care (EAPC OnLus), Milan, Italy; Erasmus University, Rotterdam, The Netherlands; University of Navarra, Navarra, Spain; Mount Sinai Medical Center, New York, USA.

We would like to specially thank Nancy Preston, Sean Hughes and Sheila Payne for their collaboration in the language editing of the article, and the whole InSup-C project for their constant assistance and revision.

\section{Funding}

The research leading to these results has received funding from the European Union's Seventh Framework Programme (FP7/2007-2013) under grant agreement $n^{\circ} 30555$.

\section{Author details}

Atlantes Research Programme, Institute for Culture and Society, University of Navarra, Campus Universitario, 31009 Pamplona, Navarra, Spain. ${ }^{2}$ Department of Anesthesiology, Pain and Palliative Medicine, Radboud University Nijmegen Medical Centre, Nijmegen, The Netherlands. ${ }^{3}$ Department of Radiation-Oncology and Palliative Medicine, University Hospital Gasthuisberg, Leuven, Belgium. ${ }^{4}$ Faculty of Medicine, Institute of Family Medicine, University of Pécs Medical School, Pécs, Hungary. ${ }^{5}$ Instituto de investigación sanitaria de Navarra (IdiSNA), Pamplona, Navarra, Spain.

\section{Received: 19 January 2016 Accepted: 4 May 2016} Published online: 13 May 2016

\section{References}

1. Palliative care for older people: Better practices. World Health Organization, Regional Office for Europe, Copenhagen, Denmark, 2011. http://www.euro. who.int/_data/assets/pdf_file/0017/143153/e95052.pdf.

2. Pascual A, Rodríguez J, García C, et al. Estrategia en cuidados paliativos del sistema nacional de salud. actualización 2010-2014. Ministerio de Sanidad, Política social e igualdad, Madrid, 2010

3. Pascual A. Estrategia en cuidados paliativos del sistema nacional de salud. Madrid: Ministerio de Sanidad y Consumo; 2007.

4. PRISMA STATEMENT. http://www.prisma-statement.org/. Accessed 15/06, 2014.

5. Van Der Eerden M, Csikos A, Busa C, et al. Experiences of patients, family and professional caregivers with integrated palliative care in europe: Protocol for an international, multicenter, prospective, mixed method study. BMC Palliat Care. 2014;13:52.

6. A policy framework for commissioning cancer services. Guidance for purchasers and providers of cancer services (calman-hine report). London: Expert Advisory Group on Cancer (EAGC); 1995.

7. Practice gudelines. http://www.agreetrust.org/resource-centre/practiceguidelines/. Updated 2014. Accessed 08/10, 2013.

8. European pathway association. http://e-p-a.org/care-pathways/. Accessed 08/10, 2013.

9. Hawker S, Payne S, Kerr C, et al. Appraising the evidence: Reviewing disparate data systematically. Qual Health Res. 2002;12(9):1284-99.

10. Emanuel L, Alexander C, Arnold RM, Bernstein R, Dart R, Dellasantina C, Dykstra L, Tulsky J. Integrating palliative care into disease management guidelines. J Palliat Med. 2004;7(6):774-83.

11. Navarro R, Valls M, Castellano V. Atención a pacientes crónicos avanzados no oncológicos con necesidad de cuidados paliativos al final de la vida en un hospital de media y larga estancia. Med Pal. 2011;18(2):54-62
12. Vicente C, Marco J, Plaza S, et al. Influencia del plan integral de cuidados paliativos de la comunidad de madrid en la actividad de una unidad de cuidados paliativos hospitalaria. Med Pal. 2011;17(6):335-341.

13. Grupo de trabajo de la Guía de Práctica Clínica sobre Cuidados Paliativos. Guía de práctica clínica sobre cuidados paliativos. Madrid: Plan Nacional para el SNS del MSC, Madrid: Agencia de Evaluación de Tecnologías Sanitarias del País Vasco; OSTEBA N; 2008. 2006/08.

14. García-Baquero MT, Carretero Y, Martínez B, et al. Borrador del plan estratégico de cuidados paliativos de la comunidad de madrid, 2010-2014. Comunidad de Madrid, Madrid, 2010

15. Sociedad Española de Cuidados Paliativos (SECPAL). Guía de Cuidados Paliativos. SECPAL, Madrid, 2010.

16. Colomer C, Palanca I, Elola J, et al. Unidad de Cuidados paliativos: estándares y recomendaciones. Madrid: Ministerio de Sanidad y Política social; 2009.

17. Agustín $M$, Arrieta J, Benites $A$, et al. Manual para el manejo del paciente en cuidados paliativos en urgencias extrahospitalarias. Barcelona: Arrow conept SL; 2011. SUMMA 112

18. Hernández J. Programa de cuidados domiciliarios en atención primaria. Toledo: Dirección General de Atención Sanitaria SESCAM; 2004.

19. Cía R, Fernández A, Boceta J, et al. Proceso asistencial integrado de cuidados paliativos, 2e. Consejería de Salud de la Junta de Andalucía, Sevilla, 2007

20. Guía de recomendaciones clínicas: Cáncer colorrectal. Dirección General de Organización de las Prestaciones Sanitarias. Siero: Consejería de Salud y Servicios Sanitarios del Principado de Asturias; 2006.

21. Cuidados paliativos. Proceso asistencial integrado araba. Arrieta A, Hernández R, Meléndez A et al. (eds). Servicio Central de Publicaciones del Gobierno Vasco, 2012.

22. Aldasoro E, Mahtani V, Sáenz De Ormijana A, et al. Necesidades en cuidados paliativos de las enfermedades no oncológicas. un estudio cualitativo desde a perspectiva de profesionales, pacientes y personas cuidadoras, Plan Nacional para el SNS del MSC. Madrid: Servicio de Tecnologías Sanitarias del País Vasco (Osteba). Informe N²0006/4; 2012.

23. Arnedillo A. Consenso sobre atención integral de las agudizaciones de la enfermedad pulmonar obstructiva crónica (ATINA-EPOC). Semerg. 2012;39:150-4.

24. Proyecto NECPAL CCOMS-ICO. Identificación y atención integral-integrada de personas con enfermedades crónicas avanzadas en servicios de salud y sociales. Gómez-Batiste X, Martínez-Muñoz M, Blay C, et al. (eds). Barcelona: Generalitat de Catalunya, Departament de Salut; 2011.

25. Rubí M, Bertrán De Lis I, Renom F, et al. Cuidados paliativos en las enfermedades respiratorias crónicas en fase avanzada. situación actual y propuesta de organización asistencial. Med Pal. 2005;12(1):39-46.

26. Alberola V, Camps C, Germa J, et al. Modelos de cuidados paliativos en pacientes con cancer. Med Pal. 2001;8(2):80-4.

27. Pascual N. Modelos de atención a pacientes oncológicos terminales en andalucía: Una mirada sociológica. Granada: Universidad de Granada; 2011.

28. Plan andaluz de cuidados paliativos. Cía R, Fernández M, Fernández A et al. (eds). Sevilla: Junta de Andalucía; 2007.

29. Estrategia de cuidados paliativos para asturias. Gago L, Palacio I, Villaría A (eds). Coruño, Oviedo: Dirección de servicios sanitarios, Subdirección de Gestión Clínica y Calidad servicio de Salud del Principado de Asturias; 2008.

30. Programa de cuidados paliativos de aragón, Amorín M, Antón A, Ara A, et al Departamento de Salud y Consumo. Zaragoza: Gobierno de Aragón; 2009.

31. López C, Zafrán E, Cuevas M, et al. Plan integral de cuidados paliativos de la comunitat valenciana 2010-2013. Valencia: Generalitat Valenciana; 2010.

32. Guía de cuidados paliativos de la comunidad de madrid. Consejería de Sanidad de la Comunidad de Madrid, Madrid, 2008.

33. Plan integral de cuidados paliativos de la comunidad autónoma de la región de murcia 2006-2009. Fernández P, Allegue J, Marín F et al. (eds) Murcia: Servicio Murciano de Salud; 2007.

34. Arrieta A, Hernández R, Meléndez A, et al. Cuidados paliativos. proceso asistencia integrado araba. Vitoria: Osakidetza-Servicio de salud vasco, Dirección Territorial de Álava- Departamento de Sanidad y Consumo; 2012.

35. Guía formativa del residente de oncología radioterápica. Miguez C (ed). Servicio Andaluz de Salud. Sevilla: Consejería de Sanidad; 2010.

36. Plan integral de atención sociosanitaria al deterioro cognitivo en extremadura (PIDEX). Castellanos F, Cid M, Duque P et al. (eds). Cáceres: Junta de Extremadura; 2007

37. Gomez Batiste X, MartinezMunoz M, Blay C, et al. Identification of people with chronic advanced diseases and need of palliative care in sociosanitary services: Elaboration of the NECPAL CCOMS-ICO(c) tool. Med Clin. 2013; 16(6):241-5. 140. 
38. Herrera E, Rocafort J, Cuervo M, et al. Primer nivel asistencial en cuidados paliativos: Evolución del contenido de la cartera de servicios de atención primaria y criterios de derivación al nivel de soporte. Med Pal. 2006;38(1):8592.

39. Gomez-Batiste X, Tuca A, Corrales E, et al. Resource consumption and costs of palliative care services in spain: A multicenter prospective study. J Pain Symptom Manage. 2006;31(6):522-32.

40. Gomez-Batiste X, Porta-Sales J, Pascual A, et al. Catalonia WHO palliative care demonstration project at 15 years (2005). J Pain Symptom Manage. 2007:33(5):584-90.

41. Manual_epidemiologico. http://www.isciii.es/ISCIII/es/contenidos/fdpublicaciones-isciii/fd-documentos/2009-0843_Manual_epidemiologico_ ultimo_23-01-10.pdf. Last accessed 9-9-2014.

42. Robertson N, Baker R, Hearnshaw $\mathrm{H}$. Changing the clinical behavior of doctors: A psychological framework. Qual Health Care. 1996;5(1):51-4.

43. van Bokhoven $M$, Kok G, van der Weijden $T$. Designing a quality improvement intervention: A systematic approach. Qual Saf Health Care. 2003;12(3):215. -216-220.

44. Quality plan for the national health system of spain. Review of activities to date and summary of future actions: Report, 2006-2010. Madrid: Ministerio de Sanidad y Política Social; 2009.

45. Gómez-Batiste X, Caja C, Espinosa J, et al. The Catalonia World health organization demonstration project for palliative care implementation: Quantitative and qualitative results at 20 years. J Pain Symptom Manage. 2012;43(4):783-94.

46. Van Beek K, Siouta N, Preston N, et al. To what degree is palliative care integrated in guidelines and pathways for adult cancer patients in Europe: a systematic literature review. BMC Palliat Care. 2016;15:26.

47. Siouta N, Van Beek K, Preston N, et al. Towards integration of palliative care in patients with chronic heart failure and chronic obstructive pulmonary disease: a systematic literature review of European guidelines and pathways. BMC Palliat Care. 2016:15:18.

48. Siouta N, Van Beek K, Van der Eerden M, et al. Integrated Palliative care in Europe: a systematic literature review of empirically-tested models in cancer and chronic disease. (in review process)

49. Sallnow $L$, Richardson $H$, Scott $M$, et al. The impact of a new public health approach to end-of-life care: A systematic review. Palliat Med. 2016;30: 200-11.

50. Weaver M, Heinze K, Bell C, Wiener L, et al. Establishing psychosocial palliative care standards for children and adolescents with cancer and their families: An integrative review. Palliat Med. 2016;30:212-23.

51. Aldridge M, Hasselaar J, Garralda E, et al. Education, implementation, and policy barriers to greater integration of palliative care: A literature review. Palliat Med. 2016;30:224-39.

52. Firn J, Preston N, Walshe C. What are the views of hospital-based generalist palliative care professionals on what facilitates or hinders collaboration with in-patient specialist palliative care teams? A systematically constructed narrative synthesis. Palliat Med. 2016:30:240-56.

53. Naveira C, Olalla MA, Miguel MP, et al. Cuidados paliativos del enfermo oncológico: guía para la gestión integrada de procesos asistenciales relacionados con el cáncer: "proyecto oncoguías" : documento de trabajo. Valladolid: Consejería de salud; 2005.

54. Herrera E, Rocafort J, Cuervo M, et al. Primary palliative care: development of the contents of the primary care services portfolio and criteria for referral according to complexity. Aten Primaria. 2006:38(2):85-92.

55. Aguilera M, Brezmes A, Escribano B, et al. Plan integral de cuidados paliativos de la Comunidad de Madrid 2005-2008. Madrid: Consejería de Sanidad y Consumo; 2005.

56. Gobierno Vasco. Plan de cuidados paliativos: atención a pacientes en la fase final de la vida; CAPV 2006-2009. Vitoria: Departamento de Sanidad; 2006.

57. Rocafort J, Herrera E, Fernández F, et al. Equipos de soporte de cuidados paliativos y dedicación de los equipos de atención primaria a pacientes en situación terminal en sus domicilios. Aten prim. 2006;38(6):316-24.

58. Ko W, Beccaro M, Miccinesi G, et al. Awareness of general practitioners concerning cancer patients' preferences for place of death: evidence from four European countries. Eur J Cancer. 2013;49(8):1967-74.

59. Alonso J, Crespo A, Sionis A, et al. Atención al paciente oncológico terminal en un distrito de Atención Primaria. Aten Primaria. 1997;9(19):483-6.
60. Canal J, Barrallat E, Vivas C, et al. Valoración de la implantación de un Programa Integral de Cuidados Paliativos. Visión retrospectiva 1995-1999. Med Pal. 2004;11(1):20-4.

61. Simó E, Faro M, Garrell I, et al. Seguimiento de los pacientes atendidos conjuntamente por un equipo de Atención Primaria y su programa de Atención Domiciliaria y Equipos de Soporte. Med Pal 2006;13(3):140-143.

62. Riera M, Barrera C, Arellano M, et al. Resultados de la evaluación de un instrumento de trabajo interdisciplinar: trayectoria clínica de la Unidad de Cuidados Paliativos. Med Pal. 2008:15(5):301-4

63. Costa G, Espinosa C, Cristófol R, et al. Demencia avanzada y cuidados paliativos, características sociodemográficas y clínicas. Med pal. 2012;19(2):53-7.

64. de Santiago A, Portela MA, Ramos L, et al. A new palliative care consultation team at the oncology department of a university hospital: an assessment of initial efficiency and effectiveness. Support Care Cancer. 2012;20(9):2199-203.

65. Alonso-Babarro A, Astray-Mochales J, Dominguez-Berjon F, et al. The association between in-patient death, utilization of hospital resources and availability of palliative home care for cancer patients. Palliat Med. 2013;27(1):68-75.

66. Vega T, Arrieta E, Lozano J, et al. Atención sanitaria paliativa y de soporte de los equipos de atención primaria en el domicilio. Gac Sanit. 2011;25(3):205-210.

67. Prades J, Borras JM. Multidisciplinary cancer care in Spain, or when the function creates the organ: qualitative interview study. BMC Public Health. 2011;11:141.

68. Colchero M, Del Yerro V. Care of terminally ill oncology patients in an urban primary care district. Aten Primaria. 2009:41(2):122-3.

69. Agra Y, Sacristán A, Pelayo M, et al. Relación de la calidad de vida con diferentes modelos de atención domiciliaria en enfermos oncológicos terminales en un área sanitaria de Madrid. Rev Esp Salud Publica. 2003;77(5): 567-579.

70. Rihuete M, Santos L. Atención integral al paciente oncológico y su familia desde una intervención multidisciplinar. Med Pal. 2005;12(1):12

\section{Submit your next manuscript to BioMed Central and we will help you at every step:}

- We accept pre-submission inquiries

- Our selector tool helps you to find the most relevant journal

- We provide round the clock customer support

- Convenient online submission

- Thorough peer review

- Inclusion in PubMed and all major indexing services

- Maximum visibility for your research

Submit your manuscript at www.biomedcentral.com/submit 